\title{
Cytoskeleton and Consciousness: An Evolutionary Based Review
}

\section{Contzen Pereira}

\section{ABSTRACT}

The fields of quantum biology and physics are now starting to unite to solve the mysteries associated with the field of evolutionary biology. One such question is the origination and propagation of consciousness which has always been ambiguous and in order to understand this concept, many theories have been proposed by several philosophers and scientists. This review paper agrees with the idea, that evolution is not a random process but hypothesizes, that its succession was managed by the expanding level of consciousness due to cell division and cell differentiation. Several theories propose that the cytoskeleton and its proteins are promoters for consciousness in the brain, which propagates by means of super-conductance. A better correlation of cytoskeletal evolution and consciousness could help solve the enigma of the origination, propagation and existence of consciousness. This review is a compilation of theories, evidences and scientific studies which intends to bring an association between propagation of consciousness and the evolution of the cytoskeleton and its proteins.

Key Words: cytoskeleton, consciousness, evolution

DOI Number: 10.14704/nq.2015.13.2.842

NeuroQuantology 2015; 2: 232-239

\section{Introduction}

Life evolved approximately 4.5 billion years ago. Lane and Martin have proposed that hydrothermal underwater vents gave rise to organic carbon containing molecules which reacted to form the building blocks of life; the nucleotides and amino acids. Proto-cells were formed from these blocks, which evolved to form cells that used proton gradients to generate energy (Lane and Martin, 2012). Some theories also propose that cellular organisms emerged as a result of micelles which are known to form by

\section{Corresponding author: Contzen Pereira}

Address: Nandadeep, 302, Tarun Bharat Soc, Chakala, Andheri (East), Mumbai 400 099, India.

Phone: : +919819642456

Fax: +912266750530

e-mail $₫$ contzen@rediffmail.com

Relevant conflicts of interest/financial disclosures: The authors declare that the research was conducted in the absence of any commercial or financial relationships that could be construed as a potential conflict of interest.

Received: 16 April 2015; Revised: 14 May 2015;

Accepted: 28 May 2015

eISSN 1303-5150 wetting and drying of lipids due to the diurnal rhythms of the sun (Torday and Rehan, 2009). Over the years, organisms have evolved based on their adaptations to the physical surroundings (Mans et al., 2004) or by means of endosymbiosis (Kutschera et al., 2005). Evolution surely did not happen by chance, so did evolution transpire because of consciousness?

The Woesian gene based revolution reclassifies life into three domains - Archae, Eubacteria and Eukaryotes which is based on the variation in the small subunit ribosomal RNA gene (Woese and Fox, 1977). The recent accumulation of DNA sequence data from numerous genes has made it clear that eukaryotic genomes are chimeric with respect to archaea and bacteria (Pühler et al., 1989; Zillig 1991; Golding and Gupta, 1995; Brown and Doolittle, 1997; Doolittle, 1996). About 540 million years ago unicellular life came into existence and these organisms began cooperating with one another 
by means of bio-films and quorum sensing; the first signs of conscious behaviour.

Eukaryotes evolved more than two billion years ago, but due to lack of fossil records it has been difficult to track the evolution of the structure and function of the sub-cellular organelles (Cavalier-Smith, 2010; El Albani et al., 2010). As per evolution, it was once thought, that the driving force for the changeover between prokaryotic to bacteria and archaea to eukaryotic, was the development of a cytoskeleton, but homologues to all the major proteins of the eukaryotic cytoskeleton have now been found in prokaryotes. Although the evolutionary relationships based on protein sequencing are not similar, there is a definite similarity in their structures and similar functions such as, maintaining cell shape and polarity which provides a strong evidence that the eukaryotic and prokaryotic cytoskeletons are truly same (Shih and Rothfield, 2006; Michie and Löwe, 2006; Briegel et al., 2006).

The cytoskeleton is a dynamic unstable structure within the cell cytoplasm and is made up of components which are constantly destroyed, renewed or newly constructed. In cells of all domains of life (archaea, bacteria, eukaryotes) a cytoskeleton is found which is composed of similar proteins. However, the structure, function and dynamic behaviour of the cytoskeleton can be very different, depending on organism and cell type (Wickstead and Gull, 2011). Cytoskeleton is made up of proteins such as tubulin and actin. All tubulins are known to have evolved from a common ancestor, which resembles the protein FtsZ (Erickson, 1995; Erickson, 2007; Lowe and Amos, 1998; Nogales et al., 1998a; Nogales et al., 1998b). Although FtsZ is a homolog of tubulin, the evolutionary distance between the two proteins is substantial and it has been suggested that an undiscovered bacteria or archaea bacteria could hold the evolutionary precursor of tubulin that could determine the true evolution of this protein.

Bio-films and quorum sensing is a conscious behaviour observed in bacteria and therefore questions the existence of consciousness in single celled organisms. Based on this characteristic several religious and scientific explanations have been put forth regarding the singularity of consciousness but with more focus on the consciousness of human brain. One such theory is the Orch-OR theory penned by Hameroff and Penrose, which claims that tubulin proteins have the possibility of evoking consciousness in the brain (Hameroff and Penrose, 2014) supported by the Bandyopadhyay coherence (BC) (Sahu et al., 2013a, Sahu et al., 2013b). The Orch OR theory is based on tubulin dipole information states which can be correlated to quantum and classical computing (Hameroff and Penrose, 2014).

Evolution of the cytoskeleton holds the key to the existence of consciousness, but is like a puzzle with many pieces missing, that have yet to be found. Evolutionary biologists are in the process of evaluating the presence of tubulin like structures and molecules in fossils and primitive organisms. No doubt there is strong evidence from their 3D structures that tubulin and FtsZ are homologous proteins (Nogales et al., 1998; Löwe and Amos, 1998), but they share only very low sequence identity, most of which is confined to the GTP-binding region (de Boer et al., 1992). This review intends to bring a correlation between the evolution of the cytoskeletal proteins and its link to the origination, propagation and existence of consciousness based on the available evolutionary theories and postulations. This correlation would help us solve the anonymity associated with several unanswered questions in the field of conscious studies pertaining to evolution.

\section{Consciousness in Biology}

Consciousness is the quality or state of awareness, of being aware of an external object or something within oneself. Consciousness is associated with the ability to process, store and act on information gathered from that external environment (Mitchell and Staretz, 2011). Consciousness was always viewed as a doubtful process by many scientists and philosophers but is now a significant topic in several areas of research. It makes an individual or organism aware of the things around in both the past and the present. Edelman has described the higher order of consciousness as "conscious of being conscious" (Edelman, 2004).

Living organisms are conscious and display this effort by the way they organize themselves in their environment. Denton in his book, on primordial emotions hypothesised that consciousness may not have emerged from the need to make an internal representation of the outside world, but rather as an extension of very 
basic primitive or primordial emotional influences (Denton, 2006). In a recently published Cambridge declaration, a group of prominent scientists formally declared that consciousness likely emerged very early in evolutionary terms, and those processes that support consciousness in humans are likely a characteristic of many living creatures (Low, 2012).

Unicellular eukaryotic ancestors of organisms like amoeba and paramecia perform complex adaptive movements and demonstrate purposeful conscious behaviour by complete utilization of the cytoskeleton. Some studies have also shown that an organism like paramecium demonstrates the capability of learning through repetitive conscious behaviour (Gelber, 1958). Amoeba also searches its food by moving on pseudopods toward its prey that they eventually surround, engulf and digest. Several types of algae are so versatile and are known to change the process how they obtain food based on available sunlight which they sense through a photoreceptor at one end of the cell. If the light is too bright, they will swim away toward more suitable lighting conditions (Mitchell and Staretz, 2011).

Consciousness in the human brain is ultrafast, and consists of discrete events at varying frequencies that have been recorded through modern science via gamma synchrony electroencephalography (EEG). Synaptic transmission and axonal transfer of nerve impulses are too slow to organize coordinated activity in large areas of the central nervous system. The transmission of nerve impulses along an axon is relatively slow and cannot explain conscious events that happen at ultra-fast speeds by means of the neural network in the human brain (Reinis et al., 2005). Several theorists have therefore proposed quantum based theories to explain consciousness e.g., the holonomic brain theory of Karl Pribram and David Bohm (Pribram, 1982) and the Orch-OR theory formulated by Stuart Hameroff and Roger Penrose (Hameroff and Penrose, 2014). These theories correlate quantum processes in relation to known physiological functions of biological organelles such as the cytoskeleton. Cytoskeleton evolution holds the secret to propagation and origination of consciousness, which cannot be defined, but exists, in every individual biological cell.

\section{Evolution of the Cytoskeleton}

The phylogenetic method classifies life into three domains -Archaea, Bacteria and Eukarya (Woese and Fox, 1977). In most cells of all domains of life, a cytoskeleton has been found and its proteins remain highly conserved. The cytoskeleton as we know is composed of proteins that can form longitudinal fibres in all organisms made up of tubulin-like proteins that are tubulin in eukaryotes and FtsZ, TubZ, RepX in prokaryotes, actin-like proteins that are actin in eukaryotes and MreB, FtsA in prokaryotes and intermediate filaments, found in eukaryotes that are lamins, keratins, vimentin, neurofilaments, desmin (Wickstead and Gull, 2011; Gunning et al., 2015). Cell division is mediated in eukaryotes by actin, but in prokaryotes usually by tubulin-like FtsZring proteins. In archaeal species belonging to the Euryarchaeota phylum, it has been found that the cell division is mediated by FtsZ protein filaments that form a constricting ring structure (Margolin, 2005).

The first evidence for tubulin homologue in bacteria was FtsZ, an essential cell division protein of Escherichia coli that bound and hydrolyzed GTP and possessed a conserved seven-residue sequence nearly identical to tubulin (de Boer et al., 1992; RayChaudhuri and Park, 1992; Mukherjee et al., 1993). It has been suggested that the three tubulins diverged from a single ancestral FtsZ (Kirk-mason et al., 1988; Katiyar and Edlind, 1994; Edlind et al., 1996) with the archezoal tubulin data restricted to beta and gamma tubulins found in few protist lineages (Lai et al., 1988; Sanchez et al., 1994). Phylogenetic analysis of alpha-tubulin genes from diplomonads, microsporadia, parabasalia and heterolobosea provides convincing evidence that alpha and beta tubulins diverged before the formation of the eukaryotic groups (Keeling and Doolittle, 1996).

Bacterial FtsZs are $40-50 \%$ identical in sequence even across very divergent species while archaeal FtsZs show a similar level of identity to each other and to bacterial FtsZs (Vaughan et al., 2004). For MreB and actin, bacterial MreBs are generally about 40\% identical in sequence even across diverse species, similar to FtsZ (Erickson, 2007). FtsZ is both widely distributed and highly conserved and its presence in most lineages of bacteria is indicative of it being an ancient protein. FtsZ as discussed earlier is widespread among bacteria it shows the formation of a basket-like structure, as in the case 
of epixenosomes (Petroni et al., 2000). Epixenosomes are marine symbionts classified under bacteria that appear to have unique morphological features among prokaryotes. Their complex structure involves the overproduction of a protein that is similar to tubulin inhibitors which also binds to tubulin antibodies and is organized in stable tubular structures (Rosati et al., 1993).

Cell shape and structure is an important property of the cell and this function is maintained by the cytoskeletal network. Microtubules are hollow cylinders about $23 \mathrm{~nm}$ in diameter, most comprising of 13 proto filaments that are polymers of alpha and beta tubulin. In nine triplet sets they form the centrioles, and in nine doublets with two additional microtubules they form cilia and flagella. Jenkins and team isolated two tubulin-like genes (bacterial tubulin a (BtubA) and bacterial tubulin b (BtubB) from bacteria of the genus Prosthecobacter (Division Verrucomicrobia). These Prosthecobacter tubulins were monomeric and unlike eukaryotic tubulins, but forms dimmers (Jenkins et al., 2002). In contrast to all other prokaryotic tubulins, BtubA/BtubB can form tubules by 5 proto filaments instead of 13 , as observed in eukaryotes (Pilhofer et al., 2011) and there is enough proof that both may have been acquired by horizontal gene transfer from eukaryotes confirming that the most likely ancestor of eukaryotic tubulins as of now remains to be FtsZ (Jékely, 2014).

The self-organizing properties of the cytoskeleton presumably evolved very early. The eukaryotic cytoskeleton appears to have evolved from ancestral precursors related to prokaryotic FtsZ and MreB. Many eukaryotes possess FtsZ genes of prokaryotic origin inherited from the endosymbiotic ancestors of mitochondria and chloroplasts. Through evolution, FtsZ developed the capability to assemble into proto- filaments. These proto-filaments demonstrated rigidity and this mechanical property might have been adopted outside of the $\mathrm{Z}$ ring support. The evolution of the cytoskeleton is a key step to propagation of consciousness which was more of a survival advantage which could provide support in anticipating threats and strategic opportunities as key genes for the brain evolved much before (Wickramasinghe, 2011).

Based on the several findings in the field of evolutionary biology, it is evident that protein

eISSN 1303-5150 and similar proteins that constitute the cytoskeleton have existed from the very beginning of life, and though not comparable to the current forms, have given rise to similar structures by maintaining their purpose of existence. Consciousness can't be measured or observed but if we assume that consciousness occurs through the cytoskeleton network, we should state that a potential consciousness can be expressed by any cell containing a cytoskeleton network and in all animal species.

\section{Cytoskeleton as a Propagator for Consciousness}

Evolution of cells can be explained in a simplified manner as the origination of small bi-lipid membrane vesicles filled with water and complex sets of organic chemicals which resembled simple non-living cells. Perhaps 3.8 billion years ago, unexplained activities created the mechanisms for replication that allowed the emergence of simple single-cell life which was also the origination of consciousness. For next billion years, these conscious single cell organisms evolved in complexity e.g. endosymbiosis of mitochondria and chloroplasts, creation of the nucleus, etc. A proof of conscious behaviour of single celled organism is the formation loose cooperative communities called as bio films by bacteria (Davey and O'toole, 2000). This characteristic was also found in fossilized cyanobacteria that evolved more than three billion years ago and is visible today as the beautiful patterns of the stromatolite fossil reefs in Australia (McNamara and Awramik, 1992).

FtsZ originated in the common ancestor and was passed to bacteria and euryarchaea, where now it is used in almost all modern day species. This makes it likely that FtsZ was also used for division in the earliest eukaryotic line, whatever may have been its origin. Even though it doesn't look so simple, based on the evidences it can be confirmed that consciousness prevailed from the beginning of life, as the cellular structures were formed as part of the formation and re-formation of matter in the universe. At a cellular level, this information processing machinery of life was stored in a complex network of thousands of genes and geneexpression control pathways that dynamically adapted the cell's function to its environment.

There are several studies that link the cytoskeleton to cognitive processes (e.g., Dayhoff 
et al., 1994; Hameroff and Penrose, 1996; 2014). Based on these postulations and hypothesis, there are several theoretical models and simulations which suggest that conformational states of tubulins within microtubular lattices are influenced by quantum events (Hameroff and Watt, 1982; Rasmussen et al., 1990; Tuszynski et al., 1995). Some models also suggest that quantum coherence is the key promoter for microtubular computation (Jibu et al., 1995) and which strongly supports the existence and propagation of consciousness within the cytoskeletal network of the cells.

In evolution, it has been proposed that quantum coherence might have emerged in eukaryotic microtubule assemblies, by means of the Fröhlich mechanism, as a by-product of coordinated dynamics and biochemical energy (Fröhlich, 1968; 1970; 1975). This theory should also hold true for the ancestral precursors in the evolution of the cytoskeleton wherein, quantum coherence would have been an intrinsic property of prokaryotic FtsZ and MreB, which helped propagate consciousness in primitive cells. Consciousness cannot be differentiated into types, because even single celled organisms have demonstrated highly conscious activities, such as bio-film formation and quorum sensing that were used to avoid threatening situations and a mode to find food. Quorum sensing is an important conscious activity observed in bacteria that helps the bacteria to coordinate certain behaviours such as bio-film formation, virulence and antibiotic resistance depending on the local density of the bacterial population (Miller and Bassler, 2001).

Microtubules are composed of tubulin dimers which are now considered as biological electric dipoles that demonstrate a Fröhlich coherent state (Wu and Austin, 1977; 1978; 1981). These dipole states mediate computation and entanglement as electric or magnetic, which was demonstrated by "Bandyopadhyay Coherence" at alternating currents of gigahertz, megahertz and kilohertz frequencies at room temperature (Sahu et al., 2013a; Sahu et al., 2013b). Ultra-fast networking in gigahertz, megahertz and kilohertz frequencies is definitely required for the propagation of consciousness as it cannot be managed by the conventional neuronal networking process.

Over the years, consciousness has not evolved but it has only increased with cellular process such as cell division and cell differentiation, which actually are also products of cellular consciousness. Evolution has permitted a wide range of medium for communication between two living organisms varying from information transfer by means of chemical, direct contact or through specialized receptors. The human brain is a multicellular organ which is a product of consciousness; made up of highly differentiated cells that propagate higher orders of consciousness through the wellorganized cytoskeletal network. Penrose and Hameroff claim that constant formation and reformation of tubulin states in the cytoskeleton of the neural cells are governed by quantum mechanical effects that create a conscious moment (Hameroff, 1998a; 1998b).

Cytoskeleton evolution is a critical marker for the origination, formation and propagation of consciousness and its existence from the very beginning of life clearly demonstrates the correlation, whether in unicellular or multicellular organisms. Cell division and differentiation are offshoots of consciousness, and it is because of this trait, life and its systems evolved to accommodate higher volumes of consciousness. Another reason for enhanced consciousnesses was species survivability which gave the organism the opportunity to mutate to avoid extinction.

\section{Were primitive organisms conscious?}

The cytoskeleton has always been considered as a unique feature to eukaryotes, which changed with the discovery that bacteria too possess homologues of both tubulin (de Boer et al., 1992; RayChaudhuri and Park, 1992; Mukherjee et al., 1993) and actin (Bork et al., 1992). Cytoskeleton has been linked to several human based cognitive processes based on quantum coherence in tubulin dimers and therefore should have also been an intrinsic property of prokaryotic FtsZ and MreB which are homologs of tubulin and actin. Bio-film formation, quorum sensing in bacteria and isotopic behavioural signatures observed in stromatolites confirmed the existence of highly conscious activities performed by primitive organisms which proves, that consciousness originated with the evolution of the cytoskeleton.

Some studies have also shown that an organism like paramecium demonstrates the capability of learning through repetitive 
conscious behaviour (Gelber, 1958). These organisms have no synapses or neural networks and hence they rely on their cytoskeleton for sensation, locomotion and information processing. Chemotaxis observed in amoeboid cells is a phenomenon that depends on amoeboid movements which are managed by the pseudopods (Taylor and Condeelis, 1979), and investigations of cellular aspects of chemotaxis in amoebae have focused on the conscious behaviour of pseudopods (Swanson and Taylor, 1982; Varnum-Finney et al., 1987; Stossel, 1989). Through these examples it can be confirmed that consciousness existed from the very beginning and is not confined to the neural network.

The existence of consciousness in primitive organisms based on the evidences gathered in this paper, supports the Cambridge declaration that occurred on July 7, 2012, at the first annual Francis Crick memorial conference on consciousness where it was confirmed by a group of prominent scientists formally declared in a document entitled the "Cambridge Declaration on Consciousness in Non-Human Animals" that the neurobiological structures needed to support consciousness are not uniquely human (Low, 2012). This declaration essentially states that the capacity for consciousness likely emerged very early in evolutionary terms, and those processes that support consciousness in humans are likely characteristic of many living creatures.

\section{Conclusion}

Consciousness cannot be reduced to just a neuronal activity but is a unique feature that was formed with the origin of life. Based on the available evolutionary information we can conclude that consciousness existed from the very beginning and has been propagating by means of the cytoskeletal network of the cell via techniques that we call quantum computing. Cytoskeletal structures have provided internal organization, cellular division, variation in cell shape, separation of chromosomes and cellular differentiation. An essential factor in evolution; cellular differentiation resulted in emergence of specialized tissues and organs from groups of cells that began to differ and develop specific and complementary form and functions through means of consciousness. Consciousness is our primary reality and through it, we perceive ourselves and our environment; we plan and accomplish our actions, evaluate them, think about them and record them.

Quantum consciousness holds an important place in understanding the functioning and propagation of consciousness. The search for a common ancestor still prevails but the use of phylogenetic relationships has helped us narrow down this search considerably. It is only through the consensual regularities of consciousness that we would come to know the real world and discover its natural and scientific properties. The evolution of the brain and the nerve cells was a result of cellular consciousness; a major progress in cytoskeletal evolution for the propagation of consciousness. The human brain is currently evolving to demonstrate a higher level of intelligence by means of propagation and full utilization of every bit of consciousness in the nerve cell, which is a possibility only by means of conscious perceiving that, would provide humans with a new degree of freedom that gives them the ability of controlling oneself. 


\section{References}

Bork P, Sander C and Valencia A. An ATPase domain common to prokaryotic cell cycle proteins, sugar kinases, actin, and hsp70 heat shock proteins. Proc Natl Acad Sci USA 1992; 89:7290-7294.

Briegel A, Dias DP, Li Z, Jensen RB, Frangakis AS and Jensen GJ. Multiple large filament bundles observed in Caulobacter crescentus by electron cryotomography. Mol Microbiol 2006; 62 (1): 5-14.

Brown JR and Doolittle WF. Archaea and the prokaryotetoeukaryote transition. Micro Mol Biol Rev 1997; 61: 456502.

Davey ME and O'toole GA. Microbial Biofilms: from Ecology to Molecular Genetics. Microbiol Mol Biol Rev 2000; 64(4): 847-867.

Dayhoff JE, Hameroff S, Lahoz-Beltra R and Swenberg CE. Cytoskeletal involvement in neuronal learning: a review. Eur Biophys J 1994; 23:79-93.

de Boer P, Crossley R, Rothfield L. The essential bacterial cell division protein FtsZ is a GTPase. Nature (London) 1992; 359:254-256.

Denton DA. The Primordial Emotions: The Dawning of Consciousness Oxford Univ Press, Oxford, UK. 2006.

Doolittle WF. Some aspects of the biology of cells and their possible evolutionary significance, in Evolution of Microbial Life (Roberts, D.M. et al., eds). Cambridge University Press, 1996; pp. 1-22,

Edelman G. Wider than the sky: The phenomenal gift of consciousness: Yale Univ Pr. New Haven, CN, USA. 2004.

Edlind TD, Li J, Visvesvara GS, Vodkin MH, McLaughlin GL and Katiyar SK. Phylogenetic analysis of b-tubulin sequences from amitochondrial protozoa. Mol Phylogent Evol 1996; 5:359-367.

El Albani A, Bengtson S, Canfield DE, Bekker A, Macchiarelli R, Mazurier A, Hammarlund EU, Boulvais P, Dupuy JJ, Fontaine C. et al. Large colonial organisms with coordinated growth in oxygenated environments $2.1 \mathrm{Gyr}$ ago. Nature 2010; 466:100-104.

Erickson HP. FtsZ, a prokaryotic homolog of tubulin? Cell 1995; 80: 367-370.

Fröhlich $\mathrm{H}$. Long-range coherence and energy storage in biological systems. Int J Quantum Chem 1968; 2:641649.

Fröhlich H. Long range coherence and the actions of enzymes. Nature 1970; 228:1093.

Fröhlich H. The extraordinary dielectric properties of biological materials and the action of enzymes. Proc Natl Acad Sci 1975; 72:4211-4215.

Gelber B. Retention in Paramecium aurelia. J Comp Physiol Psych 1958; 51:110-115.

Golding GB and Gupta RS. Protein-based phylogenies support a chimeric origin for the eukaryotic genome, Mol Biol Evol 1995; 12: 1-6.

Gunning PW, Ghoshdastider U, Whitaker S, Popp D, Robinson RC. The evolution of compositionally and functionally distinct actin filaments. J Cell Sci 2015. doi: $10.1242 /$ jcs. 165563.

Hameroff S. Quantum computation in brain microtubules? The Penrose-Hameroff Orch OR model of consciousness. Phil Trans R Soc Lond A 1998a; 356: 1869-1896.

Hameroff S. Quantum Computation in Brain Microtubules? The Penrose-Hameroff "Orch Or" Model of Consciousness. Phil Tran Math Phys Eng Sci 1998b; 356(1743): 1869-1896.

Hameroff SR and Penrose R. Orchestrated reduction of quantum coherence in brain microtubules: A model for consciousness. In: Toward a Science of Consciousness eISSN 1303-5150
The First Tucson Discussions and Debates, S.R. Hameroff, A. Kaszniak and A.C. Scott (eds.), MIT Press. 1996.

Hameroff S and Penrose R. Consciousness in the universe. A review of the 'Orch OR' theory. Phys Life Rev 2014; 11: 39-78.

Hameroff SR and Watt RC. Information processing in microtubules. J Theor Biol 1982; 98: 549-561.

Jékely G. Origin and Evolution of the Self-Organizing Cytoskeleton in the Network of Eukaryotic Organelles. Cold Spring Harb Perspect Biol 2014; 2: 6(9).

Jenkins C, Samudrala R, Anderson I, Hedlund BP, Petroni G, Michailova N, Pinel N, Overbeek R, Rosati G and Staley JT. Genes for the cytoskeletal protein tubulin in the bacterial genus Prosthecobacter. PNAS 2002; 99(26): 1704917054.

Jibu M, Pribram KH and Yasue K.From conscious experience to memory storage and retrieval: The role of quantum brain dynamics and Bose condensation of evansecent photons. Int J Mod Physics B 1996; (13\&14):1735-1754.

Katiyar SK and Edlind TD. Beta-tubulin genes of Trichomonas vaginalis. Mol Biochem Parasitol 1994; 64:33- 42.

Keeling PJ and Doolittle WF. Alpha-tubulin from early diverging eukaryotic lineages and the evolution of the tubulin family. Mol Biol Evol, 1996; 13 (10): 1297 1305.

King CC. Cosmological Foundations of Consciousness. J Cosmo 2011; 14

Kirk-Mason KE, Turner MJ and Chakraborty FR. Cloning and sequence of beta-tubulin cDNA from Giardia lamblia. Nucleic Acids Res 1988; 16:2733.

Koch C. The Quest for Consciousness. Englewood CO: Roberts \& Company, 2004.

Kutschera U and Niklas KJ. Endosymbiosis, cell evolution, and speciation. Theory Biosci 2005; 124:1-24.

Lai EY, Remillard SI and Fulton C. The alphatubulin gene family expressed during cell differentiation in Nuegleria gruberi. J Cell Biol 1988; 106:2035-2046.

Lane $\mathrm{N}$ and Martin WF. The Origin of Membrane Bioenergetics. Cell 2012; 151: 1406-1416.

Low P. Consciousness in human and non-human animals. The Francis Crick Memorial Conference. Eds Panksepp J, et al. (Cambridge, UK). 2012. Available at http:// fcmconference.org/img/CambridgeDeclarationOnConsci ousness.pdf. Accessed April 10, 2015.

Mans BJ, Anantharaman V, Aravind L and Koonin EV. Comparative genomics, evolution and origins of the nuclear envelope and nuclear pore complex. Cell Cycle 2004; 3: 1612-1637.

Margolin W. FtsZ and the division of prokaryotic cells and organelles. Nat Rev Mol Cell Biol 2005; 6:862-871.

McNamara KJ and Awramik SM. Stromatolites: a key to understanding the early evolution of life. Sci Prog 1992; 76: 345-364.

Michie KA and Löwe J. Dynamic filaments of the bacterial cytoskeleton. Annu Rev Biochem 2006; 75: 467-92.

Miller MB and Bassler BL. Quorum sensing in bacteria. Annu Rev Microbiol 2001; 55 (1): 165-99.

Mitchell ED and Staretz R.The Quantum Hologram and the Nature of Consciousness. J Cosmo 2011; 14.

Mukherjee A, Dai K and Lutkenhaus J. Escherichia coli cell division protein FtsZ is a guanine nucleotide binding protein. Proc Natl Acad Sci USA 1993; 90:1053-1057.

Nogales E, Wolf SG and Downing KH. Structure of the alpha beta tubulin dimer by electron crystallography. Nature 1998a; 391:199-203. 
Nogales E, Downing KH, Amos LA and Löwe J.Tubulin and FtsZ form a distinct family of GTPases. Nat Struct Biol 1998b; 5: 451-458.

Petroni G, Spring S, Schleifer K, Verni F and Rosati G. Defensive extrusive ectosymbionts of Euplotidium (Ciliophora) that contain microtubule-like structures are bacteria related to Verrucomicrobia. PNAS 2000; 97(4): 1813-1817.

Pilhofer M, Ladinsky MS, McDowall AW, Petroni G and Jensen GJ. Microtubules in Bacteria: Ancient Tubulins Build a Five-Protofilament Homolog of the Eukaryotic Cytoskeleton. PLoS Biol 2011; 9(12).

Pribram K. Holographic Brain. In the Omni Interviews, (Ed.) P. Weintraub, New York: Ticknor \& Fields, 1982; pp.3334.

Pühler G, Leffers H, Gropp F, Palm P, Klenk HP, Lottspeich F, Garrett RA and Zillig W. Archaebacterial DNA-dependent RNA polymerases testify to the evolution of the eukaryotic nuclear genome. Proc Natl Acad Sci USA 1989; 86: 4569-4573.

Rasmussen S, Karampurwala H, Vaidyanath R, Jensen KS and Hameroff S. Computational connectionism within neurons: A model of cytoskeletal automata subserving neural networks. Physica D 1990; 42:428-449.

RayChaudhuri D and Park JT. Escherichia coli cell-division gene ftsZ encodes a novel GTP-binding protein. Nature 1992; 359:251-254.

Reinis S, Holub RF and Smrz P. A quantum hypothesis of brain function and consciousness. Ceskoslovenska fyziologie 2005; 54; 26 - 31.

Rosati G, Lenz, P and Verni F. Epixenosomes, peculiar epibionts of the ciliated protozoon Euplotidium itoi: what kind of organisms are they? Micron 1993; 24, 465471.

Russell SJ and Norvig P. Ch. 26: Philosophical foundations. Artificial Intelligence: A Modern Approach. Prentice Hall 2010.

Sahu S, Ghosh S, Ghosh B, Aswani K, Hirata K, Fujita D. et al. Atomic water channel controlling remarkable properties of a single brain microtubule: correlating single protein to its supra molecular assembly. Biosens Bioelectron 2013a; 47:141.

Sahu S, Ghosh S, Hirata K, Fujita D and Bandyopadhyay A. Multi level memory switching properties of a single brain microtubule. Appl Phys Lett 2013b; 102:123701.

Sanchez MA, Pea-i-Tie DA, Wirth D, Orozco E. Cloning, genomic organization and transcription of the Entamoeba histolytica alpha-tubulin-encoding gene. Gene 1994; 146:239-244.

Shih YL and Rothfield L. The bacterial cytoskeleton. Microbiol Mol Biol Rev 2006; 70 (3): 729-54.

Stossel TP. From signal to pseudopod. How cells control cytoplasmic actin assembly. J Biol Chem 1989; 264: 18261-18264.

Swanson JA and Taylor DL. Local and spatially coordinated movements in Dictyostelium discoideum amoebae during chemotaxis. Cell 1982; 28: 225-232.

Taylor DL and Condeelis JS. Cytoplasmic structure and contractility in amoeboid cells. Int Rev Cytol 1979; 56, 57-144.

Torday JS and Rehan VK. The Evolution of Cell Communication: The Road not Taken. Cell Comm Insights 2009; 2: 17-25.

Tuszynski J, Hameroff S, Sataric MV, Trpisova B and Nip MLA. Ferroelectric behavior in microtubule dipole lattices; implications for information processing, signaling and assembly/disassembly. J Theor Biol 1995; 174:371-380.

Varnum-Finney BJ, Voss E and Soll DR. Frequency and orientation of pseudopod formation of Dictyostelium discoideum amoebae chemotaxing in a spatial gradient: further evidence for a temporal mechanism. Cell Motil Cytoskel 1987; 8: 18-26.

Vaughan S, Wickstead B, Gull K and Addinall SG. Molecular evolution of FtsZ protein sequences encoded within the genomes of archaea, bacteria, and eukaryota. J Mol Evol 2004; 58:19-29.

Wickramasinghe C. The Biological Big Bang. Cosmology Science Publishers. Cambridge, 2011.

Wickstead B and Gull K. The evolution of the cytoskeleton. J Cell Biol 2011; 194 (4): 513-525.

Woese CR and Fox GE. Phylogenetic structure of the prokaryotic domain: the primary kingdoms. Proc Natl Acad Sci USA 1977; 74: 5088-5090.

Wu TM and Austin S. Bose condensation in biosystems. Phys Lett A 1977; 64:151-152, 1977.

$\mathrm{Wu}$ TM and Austin S. Coperative behavior in biological systems. Phys Lett A 1978; 65:74-76, 1978.

Wu TM and Austin S. Froehlich's model of bose condensation in biological systems. J Biol Phys 1981; 9:97-107, 1981.

Zillig W. Comparative biochemistry of Archaea and Bacteria. Curr Opin Genet Dev 1991; 1: 544-551. 